\title{
A portable powered ankle-foot orthosis for rehabilitation
}

\author{
K. Alex Shorter, PhD; ${ }^{1}$ Géza F. Kogler, PhD, CO; ${ }^{2}$ Eric Loth, PhD; ${ }^{3}$ William K. Durfee, PhD; ${ }^{4}$ Elizabeth T. \\ Hsiao-Wecksler, $\mathbf{P h D}^{\mathbf{1}^{*}}$ \\ ${ }^{1}$ Department of Mechanical Science \& Engineering, University of Illinois at Urbana-Champaign, Urbana, IL; ${ }^{2}$ School \\ of Applied Physiology, Georgia Institute of Technology, Atlanta, GA; ${ }^{3}$ Department of Mechanical and Aerospace Engi- \\ neering, University of Virginia, Charlottesville, VA; ${ }^{4}$ Department of Mechanical Engineering, University of Minnesota, \\ Minneapolis, $M N$
}

\begin{abstract}
Innovative technological advancements in the field of orthotics, such as portable powered orthotic systems, could create new treatment modalities to improve the functional outcome of rehabilitation. In this article, we present a novel portable powered ankle-foot orthosis (PPAFO) to provide untethered assistance during gait. The PPAFO provides both plantar flexor and dorsiflexor torque assistance by way of a bidirectional pneumatic rotary actuator. The system uses a portable pneumatic power source (compressed carbon dioxide bottle) and embedded electronics to control the actuation of the foot. We collected pilot experimental data from one impaired and three nondisabled subjects to demonstrate design functionality. The impaired subject had bilateral impairment of the lower legs due to cauda equina syndrome. We found that data from nondisabled walkers demonstrated the PPAFO's capability to provide correctly timed plantar flexor and dorsiflexor assistance during gait. Reduced activation of the tibialis anterior during stance and swing was also seen during assisted nondisabled walking trials. An increase in the vertical ground reaction force during the second half of stance was present during assisted trials for the impaired subject. Data from nondisabled walkers demonstrated functionality, and data from an impaired walker demonstrated the ability to provide functional plantar flexor assistance.
\end{abstract}

Key words: AFO, assistive devices, dorsiflexor weakness, foot drop, foot slap, gait, plantar flexor weakness, pneumatic, portable assistance, rehabilitation, treadmill.

\section{INTRODUCTION}

The capability for an orthosis to apply an assistive torque (e.g., dorsiflexor or plantar flexor) at the ankle joint could be significant in how patients are rehabilitated with ankle-foot orthotic systems. Conceptually, users may benefit from a portable powered ankle-foot orthosis (PPAFO) for daily use to enhance walking function, as a gait training tool in physical therapy, and/or provide prescribed external power-assist modalities for improving strength and range of motion (ROM). Impaired veterans are a motivating potential subject pool for these devices because of the high number of lower-limb battle injuries seen in recent years [1].

Current clinically prescribed ankle-foot orthoses (AFOs) use mechanical elements, such as springs, dampers,

\footnotetext{
Abbreviations: $\mathrm{AFO}=$ ankle-foot orthosis, $\mathrm{CES}=$ cauda equina syndrome, $\mathrm{CO}_{2}$ = carbon dioxide, EMG = electromyography, FES = functional electrical stimulation, GRF = ground reaction force, $\mathrm{PPAFO}=$ portable powered $\mathrm{AFO}, \mathrm{ROM}=$ range of motion, SEA = series elastic actuator, $\mathrm{TA}=$ tibialis anterior.

* Address all correspondence to Elizabeth T. Hsiao-Wecksler, Associate Professor; Department of Mechanical Science \& Engineering, University of Illinois at Urbana-Champaign, Urbana, IL 61801; 217-333-1730; fax: 217-244-6534.

Email: ethw@illinois.edu

DOI:10.1682/JRRD.2010.04.0054
} 
or the brace itself to provide functional assistance during gait by restricting unwanted motion of the foot [2-3]. A posterior leaf spring AFO, for example, provides dorsiflexion assist and plantar flexion resist through the deformation of a posterior plastic strut element in the orthosis [4]. If the user's weakness is limited to the dorsiflexors, the posterior leaf spring AFO will assist gait, but the fixed motioncontrol properties of these devices (e.g., the prevention of foot drop during swing) can impede gait during other phases of the cycle (e.g., plantar flexion during stance). Additionally, an AFO configured for motion control does not have the capability to assist plantar flexor deficiencies effectively that may be present in the user. As a result, current clinical orthotic management strategies for the loss of volitional plantar flexion are limited.

Functional electrical stimulation (FES) has been used to assist users actively during gait, whereas the traditional AFOs just described have not [5-6]. These commercially available devices use small surface electrical signals to stimulate the peroneal nerve to activate the ankle dorsiflexors to provide functional toe clearance during swing. The magnitude and timing of the stimulation can be modulated according to changing gait pattern (e.g., a change in walking speed) [7]. FES devices are compact because the user's own muscles and skeleton provide the actuation and support that AFOs traditionally provide, but the devices just described do not assist the plantar flexion during gait.

Novel powered systems have been developed to address the inherent limitations of passive AFOs by providing net power to the ankle joint for motion control and torque assistance. These powered systems are tethered to sources of power and/or computing and take two forms: (1) devices being developed for untethered daily-wear applications or (2) devices intended for laboratory research and/or clinic-based rehabilitation treatments that help persons recover from the pathology or injury [8].

The most promising of the powered systems intended for daily wear use series elastic actuators (SEAs). The SEAs used by Blaya and Herr [9] and Boehler et al. [10] consist of a direct current, motor-powered ball-screw mechanism in series with a helical spring. The computercontrolled motor provides torque assistance by driving a lead screw to vary the height of the spring to adjust rotary compliance. The SEA is mounted behind the distal shank section of a traditional AFO structural element and connected to the hinged footplate of the device. These AFOs use onboard sensors to divide the gait cycle into a series of finite states, where specific control objectives are met (e.g., maintaining toe clearance during swing). Powered systems that assist ankle-joint function also have great potential as complementary clinical rehabilitation tools, and presently, a number of researchers use them for rehabilitation, diagnostics, and training devices and in locomotion studies to perturb gait $[8,11]$. A number of groups have built fluid-powered (pneumatic or hydraulic) AFOs intended for human locomotion study and gait rehabilitation [11-15]. Electric motors have also been implemented as both direct-drive and series elements for powered AFOs [16-17].

To date, powered systems, for both daily wear and laboratory rehabilitation, have not been commercialized and exist as custom devices constructed mainly from offthe-shelf components. While these devices are capable of providing power to the ankle for both motion control and propulsive assistance, the size and power requirements of the current designs have tethered the systems to power supplies, electronics, or both. As a result, the latest powered AFOs cannot be used outside the laboratory. The creation of a portable powered orthotic system would offer a new treatment modality outside of the laboratory or clinic with the potential to greatly improve the functional outcome of the rehabilitation process.

In this article, we present a novel PPAFO to provide untethered assistance for daily in-home rehabilitation treatment (Figure 1). The key advantages of fluid power for this application are the high force/weight and force/ volume of the actuator, the capability to actuate a joint without a transmission, and the capability to transport pressurized fluid to the actuator through flexible hoses that can be placed where a shaft from a traditional motor would not reach. The high force/weight fluid power actuators that do not require transmissions and the flexibility in the placement of system components elsewhere on the body allow the weight of the device at the shank and foot to be reduced. The remainder of this article will describe the current system, characterize the performance of the system, and present results from nondisabled and impaired subjects walking with the PPAFO to demonstrate device functionality.

\section{METHODS}

The PPAFO was designed to assist impaired gait by (Figure 2)-

1. Controlling forefoot velocity at heel strike to prevent foot slap, i.e., eccentric dorsiflexor assistance. 

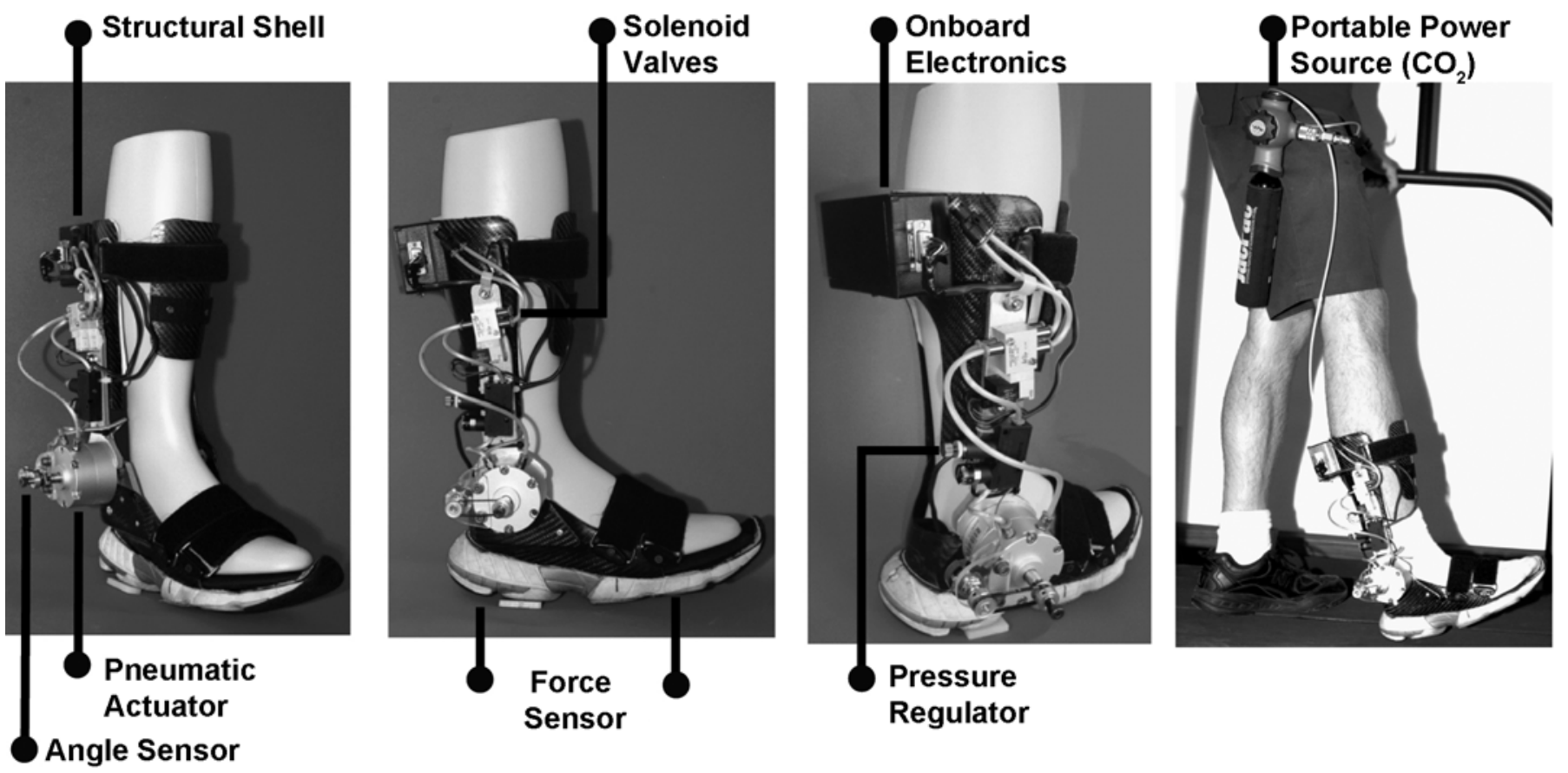

Figure 1.

First prototype of portable powered ankle-foot orthosis. Rotary actuator is powered with a compressed carbon dioxide $\left(\mathrm{CO}_{2}\right)$ bottle (far right) worn by subject on waist.

2. Allowing free ROM during stance.

3. Providing modest assistive torque for propulsion and stability at the end of stance, i.e., concentric plantar flexor assistance.

4. Supporting the foot in the neutral position during swing to prevent foot drop, i.e., concentric dorsiflexor assistance.

The "Methods" section is divided into three parts: a detailed description of the PPAFO system hardware and control scheme, empirical characterization of the system performance, and empirical testing with both nondisabled and impaired subjects to evaluate PPAFO functional performance during assistance.

\section{PPAFO System Description}

\section{PPAFO Hardware}

The PPAFO system consists of subsystems that addressed structure, actuation, sensing, and power (Figure 1). We used commercially available portable compressed liquid carbon dioxide $\left(\mathrm{CO}_{2}\right)$ bottle and pressure regulator (JacPac J-6901-91, 9 oz capacity; Supplierpipeline, Inc [Waterloo, Ontario, Canada]) to power a dualvane bidirectional rotary actuator at the ankle joint, with a maximum pressure rating of 150 psig (Figure 1)
(CRB2BW40-90D-DIM0065, SMC Corp of America; Noblesville, Indiana). The pressure regulator on the bottle modulated plantar flexor torque for propulsion assistance. Compressed gas is used safely near the human body in a number of common applications (e.g., scuba tanks, paintball guns, pneumatic hand tools). The PPAFO power source is also used to power pneumatic hand tools. To ensure user safety during operation, we used the equipment within the manufacturer's published specifications. A second pressure regulator mounted on the PPAFO (LRMA-QS-4, Festo Corp; Hauppauge, New York) modulated dorsiflexor torque for foot support during swing. The orthotic tibial and foot piece components were custom-fabricated from preimpregnated carboncomposite laminate materials over a positive model of a leg and were the structural elements of the system. The foot shell (U.S. men's size 11) incorporated a shoe-last profile that placed the heel $1.0 \mathrm{~cm}$ higher with respect to the metatarsal heads. We oriented the toe section of the footplate at a $5^{\circ}$ angle (pitch) relative to the ground to emulate late stance rollover because the foot section was rigid. A standard running shoe sole provided the interface between the foot piece and the ground. A conventional free-motion ankle hinge joint connected the foot piece to 


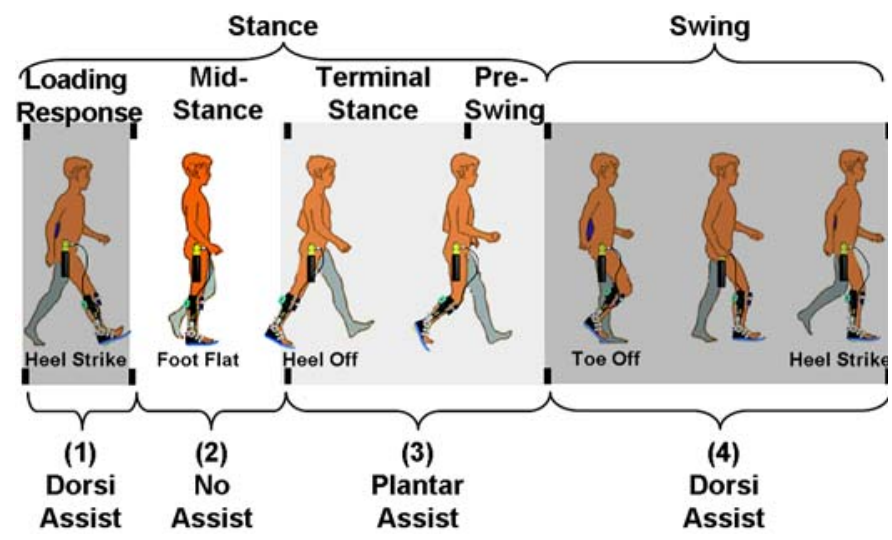

Figure 2.

Cycle was divided into multiple phases defined by functional gait tasks. portable powered ankle-foot orthosis assists gait by providing (1) assistive dorsiflexor torque at heel strike to prevent foot slap, (2) no assistive torque during midstance, (3) assistive plantar flexor torque during stance to assist propulsion, and (4) assistive dorsiflexor torque during swing to control foot motion. Source: Perry J. Gait analysis: Normal and pathological function. Thorofare (NJ): SLACK; 1992.

the tibial section on the medial aspect. Velcro straps secured the PPAFO to the leg and foot.

The direction of the torque could be switched from dorsiflexor to plantar flexor with two solenoid valves (VOVG 5V, Festo Corp; Hauppauge, New York). Switching control of the valves was selected based on specific events during the gait cycle. We determined event boundaries for these states using two force sensors (402, 0.5 in. circle; Interlink Electronics Inc [Camarillo, California]) placed on the interface of the foot section under the heel and metatarsal heads. Onboard electronics (eZ430-F2013 microcontroller, Texas Instruments; Dallas, Texas) and the portable power source allowed the PPAFO to provide untethered powered assistance.

The power source and regulator (regulator $=0.57 \mathrm{~kg}$, $\mathrm{CO}_{2}$ bottle $=0.63 \mathrm{~kg}$ ) were worn on a belt attached to the waist, separately from the structural elements of the PPAFO (AFO $=1.9 \mathrm{~kg}$ ), so the weight of the prototype (3.1 kg total) could be distributed. Working to minimize additional weight to the lower limbs is particularly important for AFOs, because accelerations at the foot are twice the individual's average walking speed [18]. Even with the weight moved to the torso, the $\sim 2 \mathrm{~kg}$ PPAFO could be expected to increase the rate of oxygen uptake $\sim 30$ percent when worn by a nondisabled adult [19] if no torque assistance were generated.

\section{PPAFO Control}

We controlled the magnitude and timing of PPAFO torque to provide the appropriate assistance during gait. Magnitudes of both the plantar flexor and dorsiflexor assistive torques were modulated by the pressure regulators. Plantar flexor assistance was set with the regulator attached to the $\mathrm{CO}_{2}$ bottle, and dorsiflexor assistance was set by a regulator fixed to the PPAFO (Figure 1). We could tune the dorsiflexor assist to a subject's individual needs by adjusting the PPAFO regulator such that the weight of the subject's relaxed foot was supported in a neutral $\left(90^{\circ}\right)$ position.

Timing of the PPAFO assistance was dictated by four regions with different functional gait requirements: (1) loading response, (2) midstance, (3) terminal stance through preswing, and (4) swing (Figure 2) [20]. During loading response, the PPAFO provided dorsiflexor assist to prevent foot slap. In midstance, the PPAFO provided no torque and allowed free ROM at the ankle joint. The structure of the device stabilized the wearer during midstance. From the beginning of terminal stance through preswing, plantar flexor torque was generated to assist with propulsion. During swing, dorsiflexor torque was generated to prevent foot drop by maintaining toe clearance.

The force sensors in the PPAFO foot piece were used to detect the event boundaries of the four regions (Figure 2). Events were detected when sensor magnitudes exceeded tuned user-specific thresholds for the heel and metatarsal sensors. Loading response began when the heel sensor threshold was exceeded at heel strike and lasted until the metatarsal sensor threshold was exceeded at foot flat. Midstance began at foot flat and continued until the heel sensor reading dropped below the threshold at heel off. Terminal stance began at heel off and continued until the metatarsal sensor dropped below threshold at the end of preswing. Swing then lasted until the following heel-strike detection. We used these specific events to direct switching control of the solenoid valves for proper assistance. The block diagram in Figure 3 illustrates the event-based control scheme used with the PPAFO.

We used a heuristic tuning scheme to determine the timing and magnitude of the PPAFO assistance for each subject. We adjusted force sensor thresholds for each subject to determine event boundaries during the gait cycle. Adjusting sensor thresholds modifies the event boundaries that are determined by the force sensors. With 


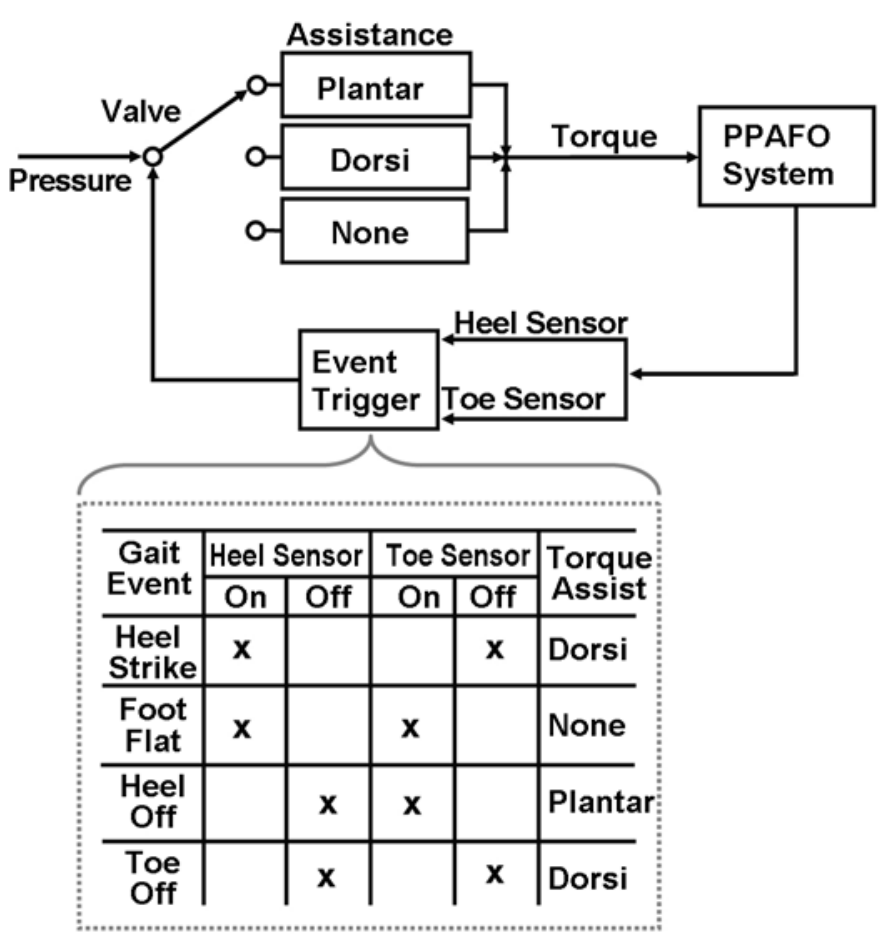

Figure 3.

Portable powered ankle-foot orthosis (PPAFO) force sensor data were used to identify current gait event. Event initiation, based on heel and toe sensor signals, determined corresponding valve configuration, which dictates type and direction of torque assistance.

the PPAFO tethered to a computer interface, the subject walked at a comfortable self-selected pace. We systematically adjusted threshold values for both heel and metatarsal force sensors using feedback from the subject to maximize user comfort. We avoided redundant triggers by maintaining a threshold large enough to exceed the noise level of the unloaded sensors. Once the sensor thresholds were determined, the values were then downloaded to the embedded microcontroller on the PPAFO, which allowed untethered operation of the device. In these trials, the subject-specific sensor thresholds did not have to be robust to changes in the subject's gait pattern because only level walking at a self-selected pace was examined.

\section{Empirical Characterization of PPAFO System Performance}

We determined performance characteristics of the PPAFO system experimentally. These performance metrics were torque generated as a function of input pressure, positional response rate of the system, energy consump- tion per actuation cycle, initial stored energy of the $\mathrm{CO}_{2}$ bottle, and continuous use duration.

The off-the-shelf PPAFO actuator was rated to a working pressure of $150 \mathrm{psig}$ and possesses a linear relationship between torque and pressure within that range in the manufacturer's documentation (CRB2BW40-90D-DIM00653, SMC Corp of America; Noblesville, Indiana). The goal of our characterization was to determine this relationship after the actuator had been integrated into the system. Torque was estimated indirectly as a function of input pressure. We used two tethered pressure transducers (4100 series, American Sensor Technology; Mt. Olive, New Jersey) to measure the pressure, $P$, in the actuator chambers to calculate assistive torque indirectly. We calculated output PPAFO torque, $\tau$, by measuring the force generated at a fixed distance from the PPAFO axis of rotation. The output torque was measured directly with a digital scale (30 lb Digital Professional, Berkley Fishing; Spirit Lake, Iowa) over a $90 \mathrm{psig}(0.62 \mathrm{MPa})$ input range. The scale was secured to the unloaded foot piece $15 \mathrm{~cm}$ away from the axis of rotation. We measured three repetitions at increasing 5 psig $(0.03 \mathrm{MPa})$ increments and then repeated for decreasing increments. We then used the averages of each repetition to determine a best-fit straight line between output torque and input pressure, such that $\tau=K_{a} P$. The coefficient $K_{a}$ represented the linear relationship between pressure and torque.

To evaluate the response rate of the PPAFO, we conducted a step-response analysis for both a loaded and an unloaded system. The loaded weight $(0.96 \mathrm{~kg})$ was selected based on the anthropometric foot weight of a nondisabled subject with body mass of $73 \mathrm{~kg}$ [21]. During the experiment, we used a $90 \mathrm{psig}(0.62 \mathrm{MPa})$ pressure to power the actuator. The foot piece was allowed to rotate through the full $90^{\circ} \mathrm{ROM}$ capacity of the rotary actuator. The 90 psig (0.62 MPa) activation level was selected because it was the largest assistive pressure used during the empirical subject testing and would respond the fastest. Position of the PPAFO foot piece was measured with an angle sensor (53 Series, Honeywell; Golden Valley, Minnesota) (Figure 1). We used five repetitions of measurements to calculate the average initiation and completion of the $90^{\circ} \mathrm{ROM}$ for both the unloaded and loaded conditions. We determined the time required to disable the system fully by pressurizing the actuator to $90 \mathrm{psig}$ $(0.62 \mathrm{MPa})$ and then timing the discharge of $\mathrm{CO}_{2}$ from the system after valve activation. Ten repetitions of the 
discharge were conducted while the PPAFO shank and footplate were maintained at a neutral position.

We determined the energy consumption of the PPAFO for an actuation cycle by measuring the $\mathrm{CO}_{2}$ consumed by the system during steady state walking of a nondisabled individual $(1.0 \mathrm{~m} / \mathrm{s})$. We examined trials at both high and low levels of assistance to span the range of energy consumption. Plantar flexor assistance at 90 psig (0.62 $\mathrm{MPa})$ and a corresponding dorsiflexor assistance at $30 \mathrm{psig}(0.21 \mathrm{MPa})$ were chosen for the high-level trial. The low-level trial was conducted with both plantar flexor and dorsiflexor assistance at $30 \mathrm{psig}$ (0.21 MPa), respectively.

To analyze the energy consumption of the PPAFO, we first collected the exhausted $\mathrm{CO}_{2}$ for each actuation cycle. We assumed that the temperature of the $\mathrm{CO}_{2}$ after the regulator was the same as the actuator $\mathrm{CO}_{2}$ exhaust to use the ideal gas law, $P_{1} V_{1}=P_{a} V_{2}$. Here, $P_{1}$ was the pressure at the regulator, $V_{1}$ was the volume of the unexpanded $\mathrm{CO}_{2}$ in the actuator, $P_{a}$ was atmospheric pressure (1.01 MPa), and $V_{2}$ was the measured volume of the exhaust gas. The mass, $m$, of the $\mathrm{CO}_{2}$ was then calculated for 20 actuation cycles during both the high and low levels of assistance, $m=P_{a} V_{2} \mu / R T$. Here, $R$ is the universal gas constant $(8.314 \mathrm{~J} / \mathrm{mol} \mathrm{K}), T$ is the gas temperature (298 K), and $\mu$ is the molecular weight of $\mathrm{CO}_{2}(0.044 \mathrm{~kg} /$ $\mathrm{mol})$. The energy consumed per cycle $\left(E_{c}\right)$ was calculated from $E_{c}=P_{1} V_{1}$ 1n $P_{1} / P_{a}$ [22]. Additionally, we calculated the initial stored energy of the bottle $\left(E_{b}\right)$ using the equation $E_{b}=m / \mu R T 1 \mathrm{n} P_{1} / P_{a}$, where $m$ is the mass of $\mathrm{CO}_{2}$ in the bottle $(0.255 \mathrm{~kg})$.

To examine the longevity of the system, we conducted a duration-of-use test. During this test, a nondisabled individual walked at the low-assistance level with the portable $\mathrm{CO}_{2}$ bottle (filled with $0.255 \mathrm{~kg}$ of $\mathrm{CO}_{2}$ ) until the final charge pressure dropped below 20 psig. We selected the low level of assistance to minimize the energy consumption and maximize the duration of use for the system.

\section{Empirical Testing of PPAFO Functional Performance During Gait}

\section{Nondisabled Subjects}

Three nondisabled male volunteer subjects (mean \pm standard deviation age $26 \pm 4 \mathrm{yr}$; height $187 \pm 7 \mathrm{~cm}$; weight $79 \pm 6 \mathrm{~kg}$ ) walked with the PPAFO on a treadmill to evaluate device functionality. Subjects had no gait impairments, had no history of significant trauma to the lower limbs or joints, and were experienced treadmill walkers.

\section{Impaired Subject}

One male volunteer subject (51 yr; height $175 \mathrm{~cm}$; weight $86 \mathrm{~kg}$ ) was recruited for the study. The subject presented with a diagnosis of cauda equina syndrome (CES) caused by spinal disk rupture that occurred during a physical therapy session. At the time of the study, the subject was 5 years postsurgery to decompress the site of injury. The primary functional motor deficit was an almost complete loss of functional plantar flexion and partial loss of sensation in the lower legs and feet with bilateral involvement. The general muscle group strength values for the impaired subject's lower limbs that were assessed with a 0 to 5.0 muscle grade scale $(0=$ no contraction; $5=$ normal strength against gravity and with resistance) gluteal $=5.0$, quadriceps $=5.0$, hamstring $=$ 4.5 , dorsiflexor $=4.5$, plantar flexor $=0.5$. The subject could walk without the use of orthoses and walking aids (i.e., cane or walker), but used bilateral off-the-shelf canvas lace-up AFOs designed to restrict movement for community ambulation. In addition to these orthoses, the subject had a pair of prefabricated carbon composite AFOs (Blue Rocker; Allard, New Jersey) that were used for bicycling activities and occasionally for community ambulation. The subject stated that he rarely used the carbon composite AFOs for walking.

\section{Subject Data Collection and Analysis (Functional Walking Analysis)}

Functionality of the device was demonstrated during treadmill walking trials. Five footwear conditions were tested: walking or running shoes and the PPAFO with no assistance, 30 psig (0.21 MPa), 50 psig $(0.34 \mathrm{MPa})$, and 90 psig (0.62 MPa) assistance. Each subject first walked in their shoes followed by randomized order of the four PPAFO conditions. For the nondisabled subject, each trial was $90 \mathrm{~s}$. For the impaired subject, each trial was $60 \mathrm{~s}$ and shoe-walking trials included no orthotic support.

Before the start of testing, we determined selfselected walking speed. For the nondisabled subjects, we determined comfortable treadmill walking speed by averaging three self-selected comfortable speeds, chosen while the subjects wore the PPAFO with no torque assist. Because we anticipated the unassisted PPAFO walking to impose the greatest walking difficulty, we selected it to 
define comfortable walking speed. Average walking speed for the three nondisabled subjects was $1.1 \mathrm{~m} / \mathrm{s}$, with a range of 0.9 to $1.3 \mathrm{~m} / \mathrm{s}$. The impaired subject's comfortable walking speed was determined while in his running shoes on the treadmill with no assistive devices on either leg. We used this walking condition because it was the impaired subject's most difficult condition. Walking speed for the impaired subject was $0.7 \mathrm{~m} / \mathrm{s}$.

During the walking trials, we collected kinematic and kinetic gait data. Subjects each wore a sleeveless top and snug-fitting shorts. We attached 45 reflective markers to the head, torso, arms, legs, and AFO. Data from nondisabled subjects were collected at the University of Illinois. We collected kinematic data using a six-camera motion analysis system sampled at $150 \mathrm{~Hz}$ (Vicon model 460; Oxford, United Kingdom). Ground reaction force (GRF) and center of pressure data for each foot were collected with a split-belt treadmill with embedded force plates sampled at 1,500 Hz (Fully Instrumented Treadmill, Bertec Corporation; Columbus, Ohio). Data from the impaired subject were collected at Georgia Institute of Technology. We collected kinematic data using a sixcamera system (Vicon model 460) sampled at $120 \mathrm{~Hz}$ and kinetic data using a split-belt custom-built treadmill with custom AMTI (Advanced Mechanical Technology, Inc; Watertown, Massachusetts) force plates sampled at 1,080 Hz [23]. During the walking trials, the impaired subject was allowed to use a forward handrail placed at chest height for stabilization, if necessary. Motion and force data for all subjects were filtered using low-pass, fourth-order, zero lag, Butterworth filters, with cutoff frequencies of $8 \mathrm{~Hz}$ and $15 \mathrm{~Hz}$, respectively. During all trials, data were also collected from the two pressure transducers and the PPAFO force sensors sampled at $30 \mathrm{~Hz}$. Surface electromyography (EMG) data (Bagnoli-16 Desktop EMG System, Delsys Inc; Boston, Massachusetts) were collected from the nondisabled subject's right tibialis anterior (TA) at 1,500 $\mathrm{Hz}$. We rectified and low-pass filtered the EMG data using a fourth-order, zero lag, Butterworth filter, with a $6 \mathrm{~Hz}$ cutoff frequency.

Following the application of the markers and EMG sensor, all subjects completed their shoe walking trial. We then conducted the randomized PPAFO trials. Because all subjects in this study had similarly sized feet and no dorsiflexor deficits, a consistent $30 \mathrm{psig}(0.21 \mathrm{MPa})$ of dorsiflexor assistance was used with all subjects during their assisted walking trials. For the nondisabled subjects, sensor thresholds for the identification of event boundaries were determined for the 30,50 , and 90 psig plantar flexor propulsive assistance after the subject's shoe walking trial. Once we had determined the sensor thresholds, we did not remove the PPAFO to reduce variability in the force sensor readings due to the strapping of the device (see "PPAFO Control" section, page 462). The subject with an impairment used a heel-walking pattern because of his impairment; consequently, he was not able to apply enough force on the metatarsal force sensor to activate the plantar flexor torque assistance. Plantar flexor torque was therefore triggered remotely for each cycle by an investigator based on visually observing the foot placement and subject's verbal commentary.

\section{RESULTS}

\section{PPAFO System Performance Characteristics}

We examined torque generation, positional system response, energy consumption of each actuation cycle, initial stored energy of the $\mathrm{CO}_{2}$ bottle, continuous duration of use, and sensor performance to evaluate PPAFO system performance. The relationship between input pressure and output torque generation was found to be represented by the slope of a best-fit straight line between the data points shown in Figure 4(a), such that $K_{a}$ $1.451 \times 10^{-5} \mathrm{~m}^{3}$. The relationship fell between the data points because of the frictional torque in the actuator. As pressure increased during the evaluation $(\times)$, static friction opposed vane motion-reducing force measurements at the scale. The opposite effect occurred as pressure was decreased from 95 psig (O), resulting in higher force measurements. Therefore, the torque difference between data points at equivalent pressures was estimated to be twice the static frictional torque of the actuator, yielding $\tau_{s}=0.45 \mathrm{Nm}$.

To evaluate the response rate of the PPAFO, we performed a step response test on both the loaded and unloaded system (Figure 4(b)). Initiation of rotation $\left(t_{1}\right)$ occurred for both the loaded and unloaded footplate $0.017 \mathrm{~s}$ after the activation signal was sent. The completion of the full $90^{\circ}$ of rotation $\left(t_{2}\right)$ of the unloaded systems was faster $\left(t_{2}=0.1 \mathrm{~s}\right)$ than the loaded system $\left(t_{2}=\right.$ $0.15 \mathrm{~s})$. The response rates of the unloaded and loaded system were thus determined to be $900 \%$ and $600 \%$, respectively. The time required to disable the system from a fully activated state was found to be $0.23 \mathrm{~s}$. 
(a)

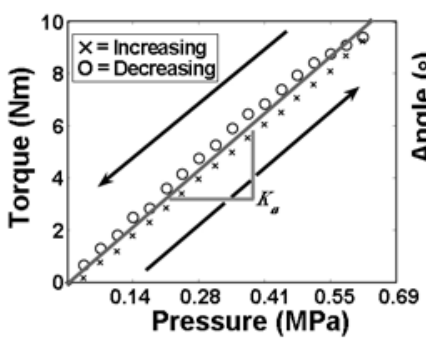

(b)

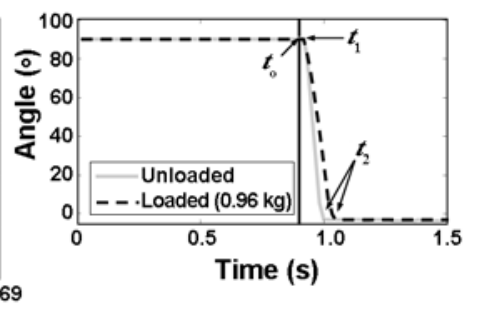

(c)

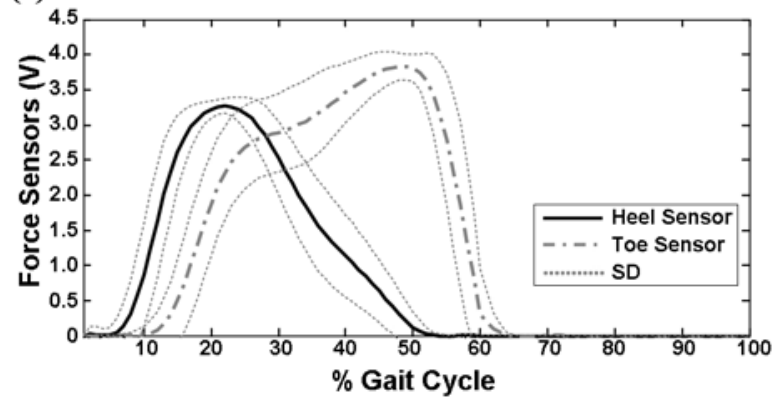

Figure 4.

(a) Experimental determination of output torque generation of portable powered ankle-foot orthosis (PPAFO) system as function of input pressure. Pressure was increased in 5 psig $(0.03 \mathrm{MPa})$ increments (denoted as $\times$ ) to 90 psig $(0.62 \mathrm{MPa})$ and then decreased in 5 psig $(0.03 \mathrm{MPa})$ increments from $90 \mathrm{psig}(0.62 \mathrm{MPa}$ ) (denoted as $\mathrm{O})$. Torque increased linearly with pressure as noted by slope $K_{a}$. (b) Positional step response for unloaded (solid line) and loaded (dashed line) PPAFO system. Valve activation $\left(t_{0}\right)$, initiation of rotation $\left(t_{1}\right)$, and cessation of rotation $\left(t_{2}\right)$ are shown in plot. (c) Average footplate force sensor data (78 cycles) from nondisabled subject. Data were normalized to percent gait cycle with toe-off occurring at $\sim 60 \%$ of cycle. $\mathrm{SD}=$ standard deviation.

We determined the energetic cost for the high and low actuation levels by measuring exhausted $\mathrm{CO}_{2}$ over 20 consecutive cycles. During the evaluation of the high actuation, the PPAFO exhausted an average of $0.166 \mathrm{~L} /$ cycle of gas. This finding resulted in an average energy consumption of $33.0 \mathrm{~J} /$ cycle. In comparison, the system at the low actuation level exhausted an average of $0.085 \mathrm{~L} /$ cycle of gas and consumed energy at an average of $9.6 \mathrm{~J} /$ cycle.

The duration of use test was conducted with 30 psig (0.21 MPa) plantar flexor and dorsiflexor assistance. The test lasted for $37.5 \mathrm{~min}$, during which the subject took 1,914 steps. We calculated initial stored energy of the bottle to be $16 \mathrm{~kJ}$. Therefore, based on continuous usage, these results translated to an energy consumption of $8.4 \mathrm{~J} /$ cycle, a rate comparable to the energetic cost analysis at the same level of assistance determined from the 20cycle $\mathrm{CO}_{2}$ exhaust experiment (9.6 J/cycle).
The capability of the PPAFO footplate force sensors to detect gait events (e.g., heel strike and toe-off) was also examined. Figure 4(c) shows the average of 78 cycles from a nondisabled walker normalized to percent gait cycle with toe-off occurring at 60 percent of the cycle. We found the gait events, used to ground truth the sensors, using GRF data from force plates in the split-belt treadmill. The force sensors detect toe-off well but with a delay of $\sim 5$ percent in the detection of heel strike. This delay in the detection of heel strike could be due to the placement of the sensors between the carbon fiber footplate and the foot of the subject instead of on the sole of the AFO.

\section{Functional Walking Results}

\section{Nondisabled Walkers}

Results from the nondisabled walking trials showed that the PPAFO provided functional torque assistance during the targeted phases of the gait cycle (Figure 5(a)): (1) dorsiflexor torque to resist foot motion during loading response, (2) free ROM early in stance, (3) modest plantar flexor assistive torque late in stance, and (4) dorsiflexor torque during swing. Sensor data were successfully used for event detection during the nondisabled walking trials (Figure 5(b)). At the highest level of plantar flexor assistance (90 psig [0.62 MPa]), the ankle kinematics were affected during both stance and swing. Dorsiflexion was reduced throughout stance, and peak plantar flexion of the joint was delayed into swing (Figure 5(c)). Neither the timing nor the magnitude of the vertical GRF was significantly affected by the PPAFO assistance (Figure 5(d)).

EMG data collected from the nondisabled walkers indicated reduced muscle activation during assistance (Figure 6). This reduction in activation level was most apparent in the TA. We reduced the muscle activation both during loading response to control of the foot motion and during swing to maintain toe clearance.

\section{Impaired Walker}

The impaired walker had retained dorsiflexor functionality but required assistance for an almost complete loss of functional plantar flexion. The subject's assisted walking trials demonstrated that the PPAFO was capable of providing functional plantar flexor assistance. However, the device was not capable of applying the plantar flexor assistance correctly during the cycle because of the impact of the subject's heel walking pattern. An investigator 

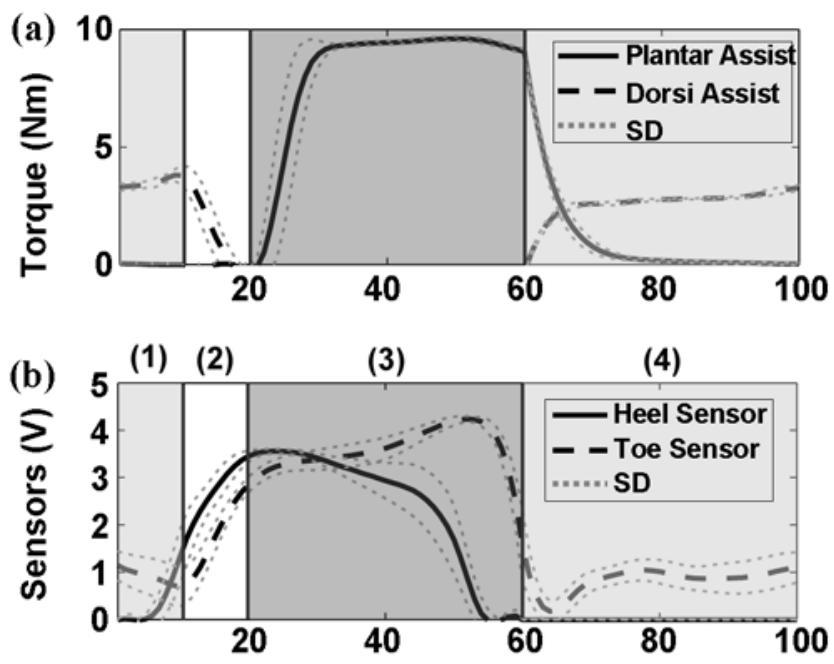

(c)

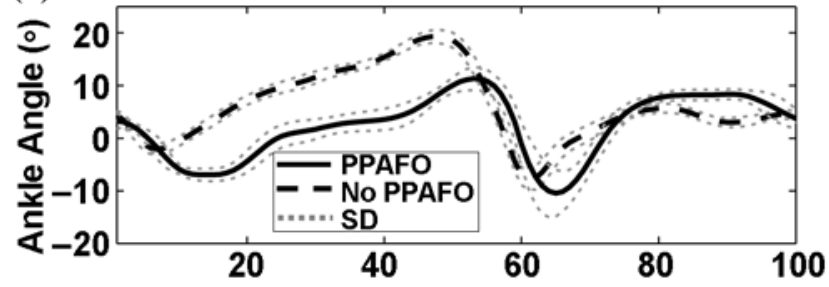

(d)

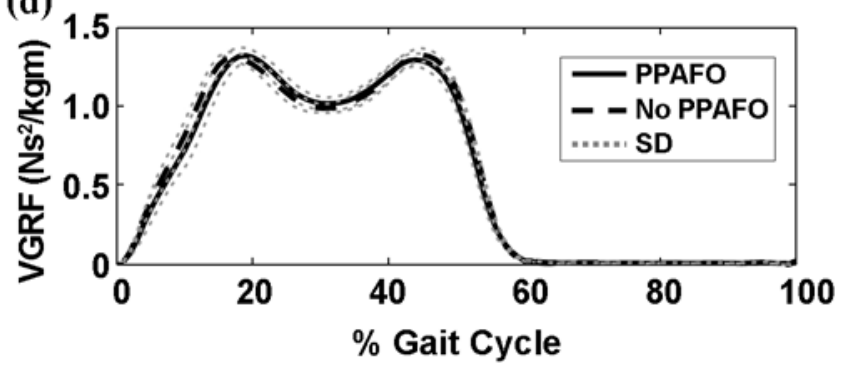

Figure 5.

Averaged data (25 gait cycles) from nondisabled walker at selfselected walking speed with peak assistive torque of $9.2 \mathrm{Nm}$ from operating pressure of $90 \mathrm{psig}(0.62 \mathrm{MPa})$. Data were normalized to stance and swing with toe-off occurring at $\sim 60 \%$ of cycle: (a) Average assistive torque produced by portable powered ankle-foot orthosis (PPAFO) during gait cycle. (b) PPAFO sensor data used for determining timing of assistance. (c) Average right ankle joint angle both with and without PPAFO. (d) Average right vertical ground reaction force (VGRF) data both with and without PPAFO. Targeted phases 1 to 4 of gait cycle are shown as (1) dorsiflexor torque to resist foot motion during loading response, (2) free range of motion early in stance, (3) modest plantar flexor assistive torque late in stance, and (4) dorsiflexor torque during swing. SD = standard deviation.

triggered plantar flexor torque remotely for each cycle, with the timing depending on investigator observation and subject comment (Figure 7(a), region 2). Dorsiflexor assist was applied in the other regions of the cycle

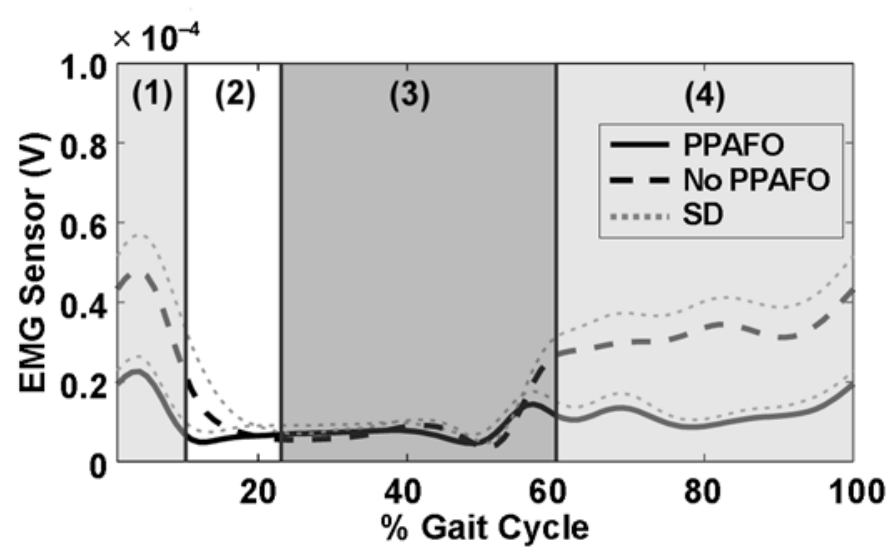

Figure 6.

Averaged data (25 gait cycles) from nondisabled walker at self-selected walking speed with (portable powered ankle-foot orthosis [PPAFO]) and without assistance (no PPAFO). Subject walked with 90 psig (0.62 MPa) plantar flexor assistance and $30 \mathrm{psig}$ (0.21 MPa) dorsiflexor assistance. Visible decrease in activation level was seen in electromyography (EMG) data from sensor located on tibialis anterior. Data were normalized to stance and swing with toe-off occurring at $\sim 60 \%$ of cycle. Targeted phases 1 to 4 of gait cycle are shown as (1) dorsiflexor torque to resist foot motion during loading response, (2) free range of motion early in stance, (3) modest plantar flexor assistive torque late in stance, and (4) dorsiflexor torque during swing. SD = standard deviation.

(Figure 7(a), regions 1 and 3). Figure 7(b) illustrates the effect the heel-walking pattern had on the PPAFO force sensors. The kinematics of the ankle joint were minimally affected by the PPAFO assistance. A small increase in dorsiflexion (solid line) at heel strike is visible in the cycle shown in Figure 7(c). The assistive capabilities of the PPAFO are most clearly illustrated in the vertical GRF data (Figure 7(d)). The second peak in the assisted vertical GRF data (solid line) indicates the presence of a push-off force during late stance, which was not present in the unassisted data (dotted line). Additionally, the subject spent a greater percentage of the cycle in stance during PPAFO assistance (70\%) than during the shoe trial (64\%).

\section{DISCUSSION}

AFOs are often an integral part of the rehabilitation process for lower-limb injuries and impairments. Current clinically available AFO technology is passive and is limited by its inability to actively modulate the assistance that the brace provides during gait. Powered orthoses have been developed in several university laboratories to 
provide net power to the ankle joint for motion control and torque assistance, but these systems are tethered and must remain in the laboratory.

In this article, we presented a novel PPAFO with potential applications for daily in-home rehabilitation treatment and demonstrated its functionality with nondisabled and impaired walkers. The torque generation of the system, up to $9 \mathrm{Nm}$ at $90 \mathrm{psig}(0.62 \mathrm{MPa})$, resulted in a device that supplied significant dorsiflexor assistance and modest plantar flexor assistance during gait. The loaded positional system response speed $(600 \%$ s) was fast enough to actuate the PPAFO during individual phases of gait (where cycle times are approximately $1 \mathrm{~s}$, with ankle ROM approximately $30^{\circ}$ ).

In addition to presenting and characterizing a novel untethered fluid-powered assist device, we used pilot data from subjects who were nondisabled and impaired to demonstrate the potential of the PPAFO to provide both plantar flexor and dorsiflexor assistance during gait. We demonstrated plantar flexor assistance directly using the data collected from an impaired subject. A suitable individual with impaired dorsiflexors for direct demonstration of dorsiflexor assistance was not currently available. As a substitute, EMG data from the TA of a nondisabled individual walking with PPAFO assistance was included in this article to demonstrate the effect of the device on dorsiflexor activation. The potential of this device for dorsiflexor assistance can be inferred from the reduction of TA activation observed in the data (Figure 6). The slow walking pace during the trials $(0.8 \mathrm{~m} / \mathrm{s})$ may be responsible for the lack of a distinct TA peak at toe-off [7]. The literature indicates that during slower walking speeds, muscle activation patterns may be more individualized. In a side study, this particular subject was reexamined at the original walking speed of $0.8 \mathrm{~m} / \mathrm{s}$ and at 1.5 and 2.0 times the self-selected walking pace. The data from these trials show TA activation without a distinct peak at toe-off during the $0.8 \mathrm{~m} / \mathrm{s}$ trials (similar to those data presented in Figure 6), but as the walking speed is increased, the peaks in TA activation became apparent at both toe-off and heel strike.

The results from the nondisabled walking trials also verified that the PPAFO provided appropriately timed powered assistance during gait (Figure 5(a)). PPAFO assistance did perturb ankle joint kinematics of the nondisabled subject shown in Figure 5(c). This perturbation reduced dorsiflexion during stance and greater but delayed plantar flexion during the start of swing. The
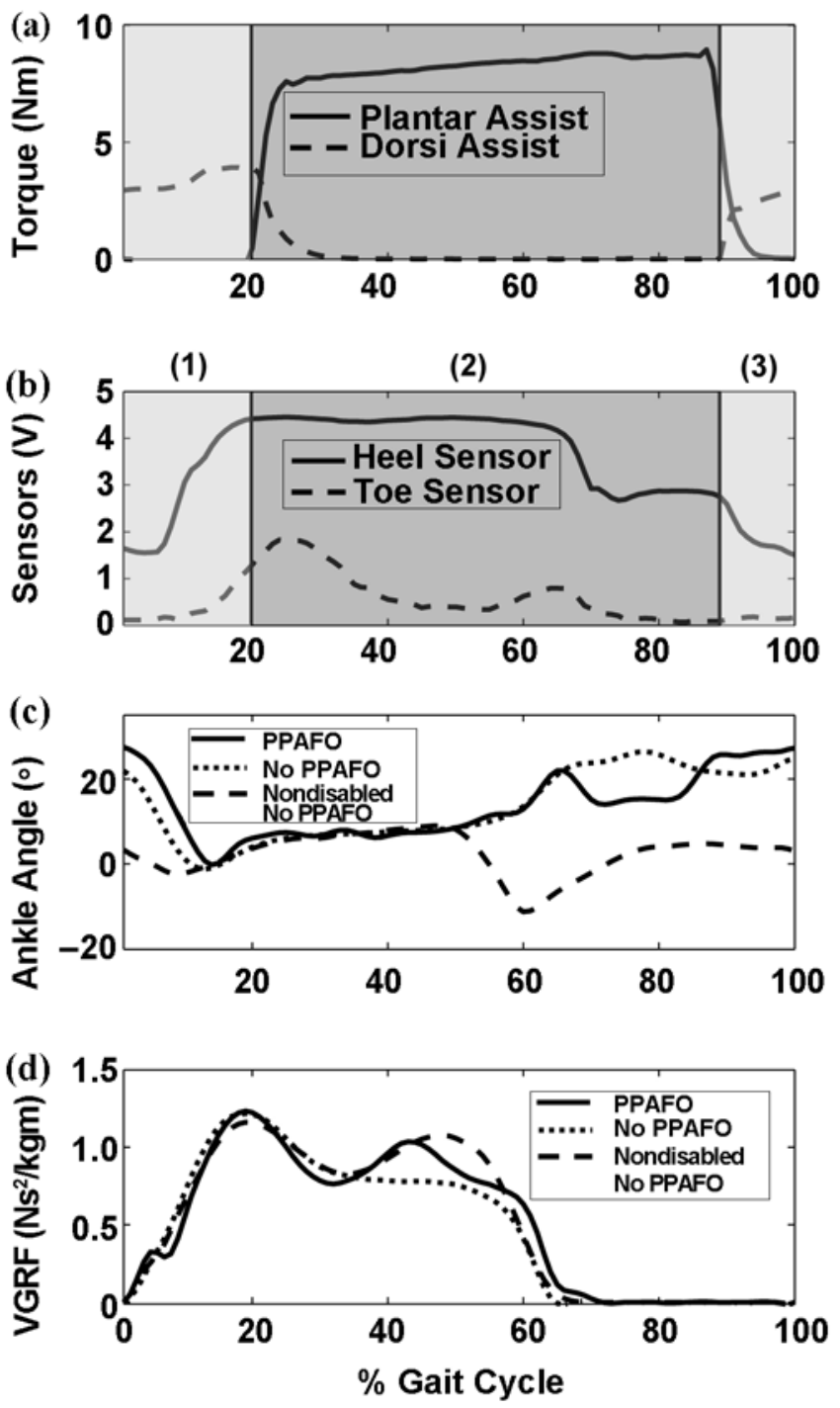

Figure 7.

One gait cycle from impaired subject with peak assistive torque of $9 \mathrm{Nm}$ from operating pressure of $90 \mathrm{psig}(0.62 \mathrm{MPa})$. Data were normalized to percent gait cycle: (a) Assistive torque produced by portable powered ankle-foot orthosis (PPAFO) during gait cycle, (b) PPAFO sensor data from heel and toe-force sensors, (c) right ankle joint angle of impaired subject both with and without PPAFO and data from nondisabled subject for comparison, (d) right vertical ground reaction force (VGRF) data of impaired subject both with and without PPFO and nondisabled subject for comparison. Phases 1 to 3 refer to gait cycle when PPAFO is providing assistance for subject.

reduced dorsiflexion could be due to the PPAFO assistance resisting motion and/or to the structure and strapping of the device reducing dorsiflexor ROM. The increased plantar flexion seen in swing could also be due to the plantar flexor assistance. The valve was closed at 
toe-off, but due to the noninstantaneous release of fluid pressure in the actuator, the magnitude of the plantar flexor assistance did not drop below the level of dorsiflexor assistance until 65 percent of the cycle (the approximate location of the peak-assisted plantar flexion).

The results from the impaired subject pilot data clearly exhibit the PPAFO's capability to provide plantar flexor assistance. The impaired subject had bilateral impairment of the lower legs due to CES but was able to walk without external aids. The subject's ability to walk without assistance permitted the direct comparison between unassisted and PPAFO-assisted walking. The subject did not use the handrail during unassisted walking but did use the rail during the assisted trial. During the assisted trial, the subject used a single hand as a guide and did not lean heavily on the rail for support. The use of the light grasp on the handrail allowed the subject an additional sensory cue to aid balance during the trial [24]. While posture may be affected by handrail walking, research has demonstrated that it may have little effect on sagittal plane kinematics [25]. Grasping the rail will affect the kinetics during walking. In our case, we believe that this effect was minimized by the subject's light grasp and the location of the rail at chest height. Because of his inability to plantar flex and invoke push off, the subject employed a heel-walking compensation strategy. Unfortunately, the tendency to bear a greater portion of stance phase loading with the heel prevented the correct triggering of the metatarsal force sensor, which was an important control element (Figure 7(b)). However, we were able to operate the plantar flexor assist manually during the walking trials.

The pilot data shown in Figure 7, along with feedback from the subject that the PPAFO provided substantive assistance, demonstrate that this device provided plantar flexor assistance sufficient for gait modification. Subject feedback during the PPAFO-assisted walking trials was positive, reporting that he could sense that his gait improved with the power assist on and could markedly distinguish the difference. We speculate that the power assist, activated at foot contact, provided resistive dorsiflexion control throughout stance. During unassisted walking, the absence of plantar flexors did not permit a heel rise during late stance, causing dorsiflexion throughout stance phase. With the loss of plantar flexion and push off, the third rocker of gait is compromised. This finding was demonstrated by the single-peak GRF data during unassisted walking (Figure 7(d), dotted line). During assisted walking, a second peak in the GRF, indi- cating push off in nondisabled gait, was present in the data (Figure 7(d), solid line). We theorize that the additional plantar flexor torque from the PPAFO acted as a dorsiflexor resist that may serve as a type of alternative rocker that transmits forces at terminal stance, creating push off. Essentially, the second rocker, which is the forward advancement of the tibia over the foot, was modified by a controlled forward progression with use of power-assisted plantar flexion. Additionally, based on the subject's perception of improved performance during gait, we speculate that the subject actively worked to maximize the efficiency of this load transmission with use of the controlled advancement of the tibia over the foot during stance. While the pilot data presented in this article demonstrate functional plantar flexor assistance, the quantification of the clinical impact that this assistance has on the end user will be examined in the future.

Innovative technological advancement in orthotic treatment strategies, such as powered orthotic systems, would be a new treatment modality that may greatly improve the functional outcomes of both civilian and combat-related injuries. Between October 2001 and May 2006, approximately 7,018 U.S. soldiers from the conflicts in Iraq and Afghanistan have suffered a severe lower-limb injury that did not result in a major lower-limb amputation [1]. This figure represents 43.7 percent of all soldiers wounded in action who did not return to duty within $72 \mathrm{~h}$ [1]. Thus, the lower limb is one of the most common regions harmed in wartime conflicts, often leading to major functional deficits that can affect joint motion and mobility. Since the impact of combat injuries to the lower limbs is so devastating, the therapeutic treatment interventions used to rehabilitate veterans are significantly important to ensure that the rehabilitation outcome of these individuals is maximized.

Although results from our study are encouraging, several important limitations must be discussed. First, we were able to demonstrate untethered assistance with nondisabled walkers, but device control issues that resulted from the impaired subject's heel walking strategy and the fit of the device to the user prevented a full demonstration of untethered functional assistance. We believe that the placement of the force sensors between the carbon fiber shell of the PPAFO and foot was partially to blame for our inability to detect gait events reliably during the testing of the impaired subject. The sensors became saturated when the PPAFO was secured to the impaired subject. As a result, the sensors did not reliably detect gait events. One could address this problem by relocating the 
sensors between the carbon fiber footplate and the sole of the PPAFO. A change in sensor location may cause readings that are not affected by the subject-specific fitting issues associated with assistive devices. We are examining this issue in future work. Second, the binary control strategy used during the nondisabled walking trails created a perturbation to the ankle joint kinematics. Control strategies that improved timing in assistance could resolve this issue.

Along these lines, we are currently working on the design of a control strategy for level walking that does not rely on direct-sensor event detection. This improved strategy will both identify relevant gait events and increase flexibility for the definition of boundaries around these events for the division of the cycle into functional objectives for the PPAFO. These functional objectives could take the form of motion control to prevent foot drop during swing, velocity control to prevent foot slap during initial stance, force control to assist with propulsion or stability during stance, or a combination of the three. The design of feedback controllers to meet these functional objectives will also improve the performance and robustness of the assistance to changes in gait pattern.

Third, we demonstrated the system's capability to assist a functional plantar flexor deficit, but we were not able to demonstrate functional dorsiflexor assistance since all subjects had functional dorsiflexors. We inferred that the PPAFO provided dorsiflexor assistance with the TA EMG data from the nondisabled subjects, but the ability to provide dorsiflexor assistance needs to be confirmed experimentally with an impaired subject who has dorsiflexor weakness. Further recruitment and testing of multiple impaired subjects will not only address this issue but also help us to continue to develop this device into a viable rehabilitation tool.

Finally, the device was demonstrated in a controlled laboratory environment. Further development of the device design along with testing outside the laboratory will be needed before the PPAFO will be ready for use in the home for treatment and rehabilitation. Logistical issues, such as $\mathrm{CO}_{2}$ replenishment, associated with a take-home assistive device will need to be addressed. One possible solution would be to refill a set of $\mathrm{CO}_{2}$ bottles during clinical follow-up visits. During these visits, the patient's rehabilitation requirements could be reassessed, a new program with modified PPAFO assistance could be created, and the patient would leave with refilled $\mathrm{CO}_{2}$ bottles. In the near term, we are working to improve the control of the device. Additionally, work is focused on reducing the weight of the orthosis on the lower leg to $<1 \mathrm{~kg}$ through integration and codesign of PPAFO system components. The performance and efficiency of the device will also be improved through the development of compact lightweight actuators and enhanced control schemes.

\section{CONCLUSIONS}

In this article, we introduced the design of a novel portable pneumatically powered AFO. Pilot data from both nondisabled walkers and an impaired individual demonstrate device functionality and lay the groundwork for future studies with larger subject populations. While direct comparisons between the nondisabled and impaired subjects are not possible, taken as a whole, data from the two groups can be used to support the potential benefits of this device. Unlike other powered orthoses, the untethered nature of the PPAFO would allow for in-home rehabilitation use. An untethered PPAFO would provide the user with increased autonomy by increasing the extent of the rehabilitation process that could occur outside a clinical setting. The PPAFO provides portability combined with the flexibility to modulate the direction (dorsal or plantar), timing, and magnitude of assistance. Such diversity allows the orthosis to meet an individual's changing functional requirements and offers promise as a clinical tool in many arenas of the rehabilitation process.

\section{ACKNOWLEDGMENTS}

\section{Author Contributions:}

Study concept and design: K. A. Shorter, G. F. Kogler, E. Loth, W. K. Durfee, E. T. Hsiao-Wecksler.

Recruitment of study population: K. Alex Shorter, G. F. Kogler. Data collection and analysis: K. A. Shorter, G. F. Kogler,

E. T. Hsiao-Wecksler.

Drafting of manuscript: K. A. Shorter, G. F. Kogler,

E. T. Hsiao-Wecksler.

Critical revision of manuscript: G. F. Kogler, E. Loth, W. K. Durfee, E. T. Hsiao-Wecksler.

Study supervision: W. K. Durfee, G. F. Kogler, E. T. Hsiao-Wecksler. Financial Disclosures: The authors have declared that no competing interests exist.

Funding/Support: This material was based on work supported by the Center for Compact and Efficient Fluid Power, a National Science Foundation Engineering Research Center, grant 0540834. 
Additional Contributions: The authors thank Yifan (David) Li, Emily Morris, Henry Kohring, Joel Gilmer, Jason Thomas, and the investigators in the Comparative Neuromechanics Laboratory at Georgia Tech (Professor Young-Hui Chang, Megan Toney, and Jasper Yen) for their assistance.

Professor Eric Loth is now at the University of Virginia, Charlottesville, Virginia.

Institutional Review: The institutional review boards at the University of Illinois and Georgia Institute of Technology approved all procedures, and all participants gave informed consent.

Participant Follow-Up: The authors plan to inform participants of the publication of this study.

\section{REFERENCES}

1. Stansbury LG, Lalliss SJ, Branstetter JG, Bagg MR, Holcomb JB. Amputations in U.S. military personnel in the current conflicts in Afghanistan and Iraq. J Orthop Trauma. 2008;22(1):43-46. [PMID: 18176164] DOI:10.1097/BOT.0b013e31815b35aa

2. Redford JB. Orthotics etcetera. Baltimore (MD): Williams \& Wilkins; 1986.

3. Rose GK. Orthotics: Principles and practice. London (UK): William Heinemann; 1986.

4. Becker Orthopedic [Internet]. Troy (MI): Becker Orthopedic; 2003 [cited 2009]. Available from: http://www.beckerorthopedic.com/cenfab/cfp.htm.

5. Weber DJ, Stein RB, Chan KM, Loeb G, Richmond F, Rolf $\mathrm{R}$, James K, Chong SL. BIONic WalkAide for correcting foot drop. IEEE Trans Neural Syst Rehabil Eng. 2005;13(2): 242-46. [PMID: 16003906]

DOI:10.1109/TNSRE.2005.847385

6. Hausdorff JM, Ring H. Effects of a new radio frequencycontrolled neuroprosthesis on gait symmetry and rhythmicity in patients with chronic hemiparesis. American Am J Phys Med Rehabil. 2008;87(1):4-13. [PMID: 18158427] DOI:10.1097/PHM.0b013e31815e6680

7. Byrne CA, O’Keeffe DT, Donnelly AE, Lyons GM. Effect of walking speed changes on tibialis anterior EMG during healthy gait for FES envelope design in drop foot correction. J Electromyogr Kinesiol. 2007;17(5):605-16.

[PMID: 16990012]

DOI:10.1016/j.jelekin.2006.07.008

8. Krebs H, Dipietro L, Levy-Tzedek S, Fasoli S, RykmanBerland A, Zipse J, Fawcett J, Stein J, Poizner H, Lo A, Volpe B, Hogan N. A paradigm shift for rehabilitation robotics. IEEE Eng Med Biol Mag. 2008;27(4):61-70. DOI:10.1109/MEMB.2008.919498

9. Blaya JA, Herr H. Adaptive control of a variable-impedance ankle-foot orthosis to assist drop-foot gait. IEEE Trans Neural Syst Rehabil Eng. 2004;12(1):24-31.

\section{[PMID: 15068184]}

DOI:10.1109/TNSRE.2003.823266

10. Boehler AW, Hollander KW, Sugar TG, Shin D. Design, implementation, and test results of a robust control method for a powered ankle foot orthosis (AFO). Proceedings of the IEEE International Conference on Robotics and Automation; 2008 May 19-23; Pasadena, CA. Los Alamitos (CA): IEEE; 2008.

11. Ferris DP, Sawicki GS, Domingo A. Powered lower limb orthoses for gait rehabilitation. Top Spinal Cord Inj Rehabil. 2005;11(2):34-49. [PMID: 16568153]

DOI:10.1310/6GL4-UM7X-519H-9JYD

12. Norris JA, Granata KP, Mitros MR, Byrne EM, Marsh AP. Effect of augmented plantarflexion power on preferred walking speed and economy in young and older adults. Gait Posture. 2007;25(4):620-27. [PMID: 16905320] DOI:10.1016/j.gaitpost.2006.07.002

13. Noël M, Cantin B, Lambert S, Gosselin CM, Bouyer LJ. An electrohydraulic actuated ankle foot orthosis to generate force fields and to test proprioceptive reflexes during human walking. IEEE Trans Neural Syst Rehabil Eng. 2008; 16(4):390-99. [PMID: 18701385] DOI:10.1109/TNSRE.2008.926714

14. Ferris DP, Gordon KE, Sawicki GS, Peethambaran A. An improved powered ankle-foot orthosis using proportional myoelectric control. Gait Posture. 2006;23(4):425-28.

[PMID: 16098749]

DOI:10.1016/j.gaitpost.2005.05.004

15. Ferris DP, Czerniecki JM, Hannaford B. An ankle-foot orthosis powered by artificial pneumatic muscles. J Appl Biomech. 2005;21(2):189-97. [PMID: 16082019]

16. Bharadwaj K, Sugar TG, Koeneman JB, Koeneman EJ. Design of a robotic gait trainer using spring over muscle actuators for ankle stroke rehabilitation. J Biomech Eng. 2005;127(6):1009-13. [PMID: 16438241]

DOI:10.1115/1.2049333

17. Krebs HI, Hogan N. Therapeutic robotics: A technology push. Proc IEEE. 2006;94(9):1727-38.

DOI:10.1109/JPROC.2006.880721

18. Barnett SL, Bagley AM, Skinner HB. Ankle weight effect on gait: Orthotic implications. Orthopedics. 1993;16(10): 1127-31. [PMID: 8255807$]$

19. Waters RL, Mulroy S. The energy expenditure of normal and pathologic gait. Gait Posture. 1999;9(3):207-31.

[PMID: 10575082] DOI:10.1016/S0966-6362(99)00009-0

20. Perry J. Gait analysis: Normal and pathological function. Thorofare (NJ): SLACK; 1992.

21. Winter DA. Biomechanics and motor control of human movement. 3rd ed. Hoboken (NJ): John Wiley \& Sons; 2005. 
22. Cai M, Kawashima K, Kagawa T. Power assessment of flowing compressed air. J Fluids Eng. 2006;128(2):402-5. DOI:10.1115/1.2170129

23. Kram R, Griffin TM, Donelan JM, Chang YH. Force treadmill for measuring vertical and horizontal ground reaction forces. J Appl Physiol. 1998;85(2):764-69. [PMID: 9688758]

24. Dickstein R, Laufer Y. Light touch and center of mass stability during treadmill locomotion. 2004;20(1):41-47. [PMID: 15196519]

25. Siler WL, Jorgensen AL, Norris RA. Grasping the handrails during treadmill walking does not alter sagittal plane kinematics of walking. Arch Phys Med Rehabil. 1997; 78(4):393-98. [PMID: 9111459]

DOI:10.1016/S0003-9993(97)90231-8
Submitted for publication April 2, 2010. Accepted in revised form November 2, 2010.

This article and any supplementary material should be cited as follows:

Shorter KA, Kogler GF, Loth E, Durfee WK, HsiaoWecksler ET. A portable powered ankle-foot orthosis for rehabilitation. J Rehabil Res Dev. 2011;48(4):459-72. DOI:10.1682/JRRD.2010.04.0054

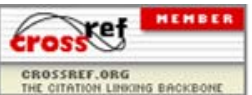

